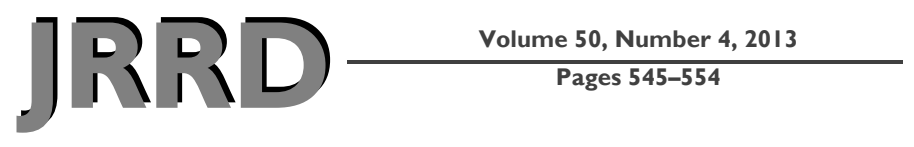

\title{
Cycling exercise to resist electrically stimulated antagonist increases oxygen uptake in males: Pilot study
}

\author{
Hiroo Matsuse, MD; ${ }^{*}$ Naoto Shiba, MD; ${ }^{1}$ Yoshio Takano, MD; ${ }^{2}$ Shin Yamada, MD ${ }^{3}$ Hiroshi Ohshima, MD; ${ }^{3}$ \\ Yoshihiko Tagawa, $\mathbf{P h D}^{4}$ \\ ${ }^{1}$ Division of Rehabilitation, Kurume University Hospital, Kurume, Fukuoka, Japan; ${ }^{2}$ Department of Physical Therapy \\ Faculty of Medical Technology, Teikyo University Fukuoka, Omuta, Fukuoka, Japan; ${ }^{3}$ Japan Aerospace Exploration \\ Agency, Tsukuba, Ibaraki, Japan; ${ }^{4}$ Department of Mechanical and Control Engineering, Kyushu Institute of Technol- \\ ogy, Kitakyushu, Fukuoka, Japan
}

\begin{abstract}
A hybrid training system (HTS) has been developed as the combined application of electrical stimulation (ES) and volitional contractions (VC), using electrically stimulated eccentric antagonist muscle contractions as a resistance to voluntary agonist muscle contractions. The purpose of the present study is to compare the metabolic cost between cycling exercise using HTS as added resistance (HTC) and unloaded cycling exercise (ULC). Twelve male subjects exercised on a leg cycle ergometer. After 5 min rest and 5 min warm up, they performed ULC for $5 \mathrm{~min}$ or HTC for $5 \mathrm{~min}$. During rest and each exercise, the steady state of oxygen uptake (VO2), carbon dioxide output (VCO2), expired ventilation (VE), respiratory exchange ratio (RER), and heart rate (HR) were measured. The values of $\mathrm{VO} 2, \mathrm{VCO} 2, \mathrm{VE}$, and $\mathrm{HR}$ during $\mathrm{HTC}$ were significantly greater than during ULC $(p<0.05)$. Relative VO2 was $16.7 \%+/-2.1 \%$ of peak VO2 during ULC, and it was $21.1 \%$ $+/-3.4 \%$ during HTC. However, there was no significant difference in RER between ULC and HTC. These findings indicate that the combined application of VC and ES could lead to a greater increase in metabolic cost. HTS seems to be applicable as a resistance of motion.
\end{abstract}

Key words: analysis of expired gas, cycling, eccentric, ergometer, exercise intensity, metabolic cost, neuromuscular electrical stimulation, rehabilitation, resistance, training, volitional contraction.

\section{INTRODUCTION}

Neuromuscular electrical stimulation (NMES) is widely used to lessen immobilization-associated muscle atrophy, to strengthen muscles, and to improve function in people with neuromuscular disabilities [1-5]. The combined application of electrical stimulation (ES) and volitional contractions (VC) is said to be more effective than ES or VC alone [6-7]. A hybrid training system (HTS) that creates resistance to the motion of a voluntary contracting agonist muscle by means of the force generated

Abbreviations: $\mathrm{ES}=$ electrical stimulation, $\mathrm{HR}=$ heart rate, HTC $=$ hybrid training system cycling exercise, $\mathrm{HTS}=$ hybrid training system, NMES = neuromuscular electrical stimulation, $\mathrm{RER}=$ respiratory exchange ratio, $\mathrm{SD}=$ standard deviation, $\mathrm{ULC}=$ unloaded cycling exercise (with volitional contractions alone), $\mathrm{VC}=$ volitional contractions, $\dot{\mathrm{V}} \mathrm{CO}_{2}=$ carbon dioxide output, $\dot{\mathrm{V} E}=$ pulmonary ventilation, $\dot{\mathrm{V}}_{2}=$ oxygen uptake, $\dot{\mathrm{V}} \mathrm{O}_{2 \text { peak }}=$ peak oxygen consumption, $\mathrm{VT}=$ ventilator threshold.

*Address all correspondence to Hiroo Matsuse, MD; Division of Rehabilitation, Kurume University Hospital, 67 Asahi-machi, Kurume, Fukuoka 830-0011, Japan; +81(0)942-31-7568; fax: +81-(0)942-46-6002.

Email: matsuse hiroh@kurume-u.ac.jp

http://dx.doi.org/10.1682/JRRD.2012.04.0067 
by its electrically stimulated antagonist has been developed (Figure 1) [8]. HTS has been shown to improve both muscle hypertrophy and strength not only in healthy young subjects [8-10], but also in elderly subjects [11]. Matsuse et al. reported that elbow flexion torque had increased about 56 percent and the proximal upper-limb muscle cross-sectional areas had increased about 10 percent by HTS over an 8-week period and those increases were better than for isotonic weight training and NMES [9]. Iwasaki et al. studied the efficacy of HTS compared with conventional weight training with 15-repetition maximum loads for increasing muscle strength around the knee at both slow and fast joint speeds ( 30 and $180^{\circ} / \mathrm{s}$ ) and reported that HTS is comparable to weight training with the exception of high-speed contractions (HTS $25 \%-28 \%$ and weight training $24 \%-33 \%$, at $30 \%$ ) [10]. In elderly subjects, HTS has been shown to produce improvements in muscle strength by about 40 percent and mass by about 10 percent that are as good as or better than those achieved with a knee flexion machine with 30 percent of the maximum voluntary contraction [11]. One of the major advantages of HTS is that it incorporates reciprocal limb movements and muscle contractions without the need for external stabilization or resistance [8-9,11]. In other words, with HTS, an unloaded exercise can be changed to a loaded exercise.

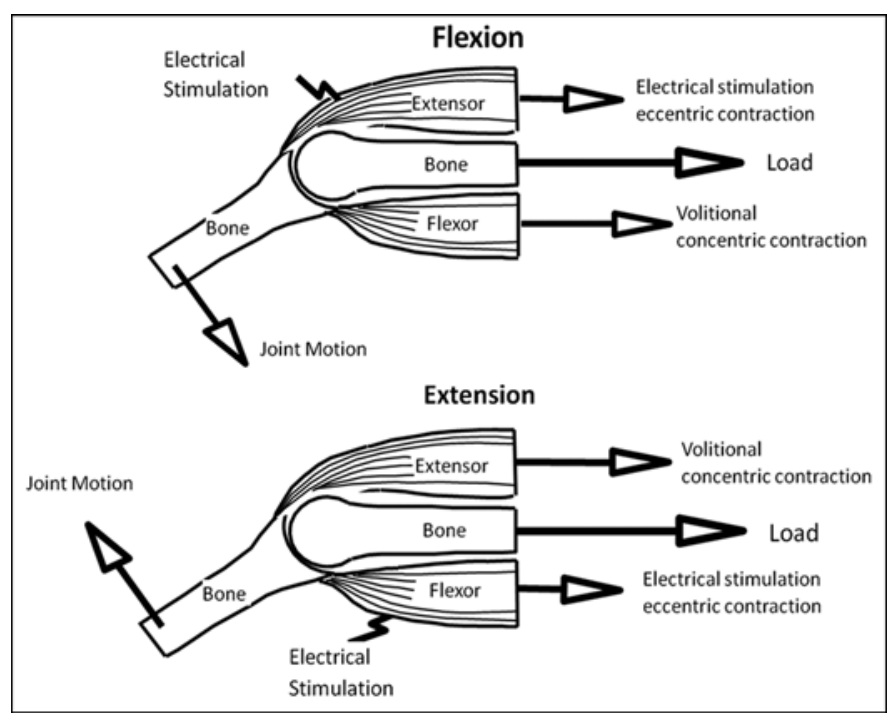

Figure 1.

Schematic model of hybrid training system. Note that both volitionally activated agonist and electrically stimulated antagonist contract during joint motion. Result is that both muscles are trained and longitudinal compressive load is placed on bone.
Oxygen uptake $\left(\mathrm{V}_{2}\right)$ is commonly used to determine the response to exercise. Furthermore, $\mathrm{V}_{2}$ during exercise shows the metabolic cost and depends on relative work rate and type of muscle action [12]. $\dot{\mathrm{VO}}_{2}$ increases as a linear function of work rate at least until the work rate exceeds the ventilator threshold (VT) [13]. In $\dot{\mathrm{VO}}_{2}$ during ES, Hayter et al. investigated the ability of muscle contractions evoked by ES with two different devices for measuring $\dot{\mathrm{VO}}_{2}$ and indicated that passive ES alone could not increase $\mathrm{VO}_{2}$ [14]. Furthermore, Hettinga and Andrews reviewed the efficacy of the various types of functional ES-assisted exercises for persons with spinal cord injury by noting the difference in $\dot{\mathrm{VO}}_{2}$ during exercise [15]. They indicated that functional ES increased $\mathrm{VO}_{2}$ during exercise and suggested that the type with higher $\dot{\mathrm{V}}_{2}$ was suited for high-intensity and high-volume exercise [15]. In this way, $\mathrm{VO}_{2}$ during ES alone has been evaluated. However the combined application of ES and VC has not been evaluated. Because cycling exercise recruits a substantial muscle mass, muscle $\mathrm{VO}_{2}$ is the primary determinant of pulmonary $\dot{\mathrm{VO}}_{2}$ [12]. Furthermore, because cycling exercise with a cycle ergometer can be coordinated at various workloads and speeds, it is widely used for $\mathrm{VO}_{2}$ kinetics using an analysis of expired gas [12,15-19]. Hooker et al. determined the effects of ES cycle training for persons with spinal cord injury by evaluating $\mathrm{VO}_{2}$ during ES cycling exercise before and after training as physiological responses [18]. Furthermore, Perrey et al. evaluated $\mathrm{V}_{2}$ during constant-load cycling exercise at $60 \mathrm{rpm}$ in comparison to the $\dot{\mathrm{VO}}_{2}$ kinetics of concentric and eccentric exercise and documented that the rate of increase in $\dot{\mathrm{V}}_{2}$ at the onset of exercise was not different between high-intensity eccentric exercise and low-intensity concentric exercise [19]. In this study, cycling exercise was used to measure $\dot{\mathrm{V}}_{2}$ during constant exercise using HTS as a resistance of motion (workload).

The purpose of the present study was to compare the metabolic cost between cycling exercise using HTS as workload (HTC) and unloaded cycling exercise with volitional contractions alone (ULC) by means of an analysis of expired gas. Electrically stimulated eccentric antagonist contractions can increase muscle strength and activity of not only antagonist but also agonist muscles and provide resistance of motion for the agonist [20]. Therefore, we hypothesized that $\dot{\mathrm{VO}}_{2}$ would increase more with the resistance of motion (workload) by electrically stimulated antagonist contractions than in ULC alone. 


\section{METHODS}

\section{Subjects}

The Ethics Committee of Kurume University and the Japan Aerospace Exploration Agency approved the clinical design of this study protocol. Following approval, informed consent was obtained from 12 healthy men (mean \pm standard deviation [SD] age $=21 \pm 1.3 \mathrm{yr}$, height $=$ $173.9 \pm 6.8 \mathrm{~cm}$, and weight $=67.2 \pm 10.8 \mathrm{~kg}$ ) who had reviewed the goals of the study and agreed to participate. Inclusion criteria included the requirements that the subjects had unremarkable musculoskeletal conditions; had no adverse medical history; and had undergone an examination for normal physical fitness, strength, sensation, and range of motion according to the criteria of the Japanese Orthopedic Association. They were also to have not participated in any regular sports activities for at least a year. These sedentary men were included because it is necessary for physical fitness and muscle strength to be standardized for evaluating the basic responses to HTS.

\section{Design}

All subjects were measured for height and body weight and performed two cardiopulmonary exercise tests in the clinical laboratory of an academic center. Environmental conditions were similar for all exercise tests $\left(21^{\circ} \mathrm{C}-24^{\circ} \mathrm{C}, 45 \%-55 \%\right.$ relative humidity). The first exercise test consisted of a ramp protocol performed on an electronically braked cycle ergometer (STB-2400, Nihon Kohden; Tokyo, Japan) in order to determine peak $\mathrm{O}_{2}$ consumption $\left(\mathrm{V}_{2 \text { peak }}\right)$ until exhaustion levels were reached [21]. On another day of the ramp exercise test, subjects were measured for gas exchange during cycling exercise by the following protocol. During the tests, gas exchange data were collected continuously with an automated breath-by-breath system (AE-300S, Minato Medical Science Co Ltd; Osaka, Japan) using the standard technique. The AE-300S consists of a microcomputer; a hot-wire flow meter; and a gas analyzer, which contains a sampling tube, filter, suction pump, $\mathrm{O}_{2}$ analyzer made by a paramagnetic $\mathrm{O}_{2}$ transducer, and an infrared $\mathrm{CO}_{2}$ analyzer. Ventilatory parameters were measured using a hotwire flow meter, and the flow meter was calibrated with a syringe of known volume $(2.0 \mathrm{~L})$. A zirconium sensor and an infrared absorption analyzer, respectively, measured $\mathrm{O}_{2}$ and $\mathrm{CO}_{2}$ concentrations. The gas analyzer was calibrated to known standard gas levels $\left(\mathrm{O}_{2} 15.16 \%, \mathrm{CO}_{2}\right.$ $5.023 \%$ ) before each test. Also heart rate (HR) (beats per minute) was continuously monitored by electrocardiogram during the tests. $\mathrm{O}_{2}, \mathrm{CO}_{2}$ output $\left(\dot{\mathrm{V} C \mathrm{CO}_{2}}\right)$, pulmonary ventilation ( $\dot{\mathrm{VE}}$ ), and respiratory exchange ratio (RER) were calculated and recorded during the following tests.

\section{Intervention and Measurements}

\section{Ramp Exercise Test}

The ramp exercise test was conducted to determine the exercise intensity of HTC as compared with $\dot{\mathrm{VO}}_{2 \text { peak }}$. After a 2 min rest sitting on the cycle ergometer, the test started at $20 \mathrm{~W}$ and the workload was increased by $20 \mathrm{~W} / \mathrm{min}$ according to the predicted physiological profile of the subjects. The pedaling cadence was kept at between 60 to $70 \mathrm{rpm}$ using a pedal frequency meter depending on the subject's preferences [21]. The ramp rate was determined so that the test duration was $8-12 \mathrm{~min}$. The exercise test was terminated when the pedal cadence could not be maintained at $60 \mathrm{rpm}$. Verbal encouragement was given during the ramp exercise test. The ventilatory threshold was determined as watts corresponding to the first breakpoint in $\mathrm{CO}_{2}$ with respect to $\mathrm{O}_{2}$ (V-slope method) [22].

\section{Cycling Exercise Test Protocol}

The cycling exercise test was performed as three tests for analysis of expired gas. The first was a rest test for 5 min. Next for the ULC test, subjects pedaled against a resistive load of $0 \mathrm{~W}$ for $10 \mathrm{~min}$ including a warm up. And for the HTC test, subjects pedaled against HTS resistance for $5 \mathrm{~min}$ after a $5 \mathrm{~min}$ warm up with $0 \mathrm{~W}$, in consideration of muscle fatigue from electrical stimulation. Pedal cadence was kept at $60 \mathrm{rpm}$ with the aid of a pedal frequency meter and a metronome. The sequence of ULC and HTC tests was performed at random, and subjects rested thoroughly (about an hour) between the ULC and HTC tests. At the onset of constant-load exercise, $\mathrm{VO}_{2}$ increases monoexponentially with a time constant to achieve a steady state within about $3 \mathrm{~min}$ in heathy individuals below the lactate threshold [23]. Therefore the values of $\mathrm{HR}$, absolute $\mathrm{VO}_{2}$ (liters per minute), relative $\dot{\mathrm{V}}_{2}$ (milliliters per kilogram per minute), $\dot{\mathrm{VCO}} 2$ (liters per minute), $\dot{\mathrm{VE}}$ (liters per minute), and RERs were averaged during the last 2 min of the steady state of each test and used for data analysis.

\section{Hybrid Training System Protocol}

For the HTC test, HTS was performed during cycling exercise on a cycle ergometer with the subject's hamstrings 
electrically stimulated as he volitionally extended his knee and his quadriceps electrically stimulated as he volitionally flexed his knee to provide motion resistance (Figure 2). During the cycling exercise, both lower limbs were stimulated in synchronization to the bending motions of the knee using HTS. The joint range of motion was set at a nearly $90^{\circ}$ arc that extended from $20^{\circ}$ to $110^{\circ}\left(0^{\circ}\right.$ indicating full knee extension) by adjusting the height of the saddle (Figure 3 ).

\section{Electrical Stimulation Protocol}

The electrical stimulation device has been described previously [8-9] and consists of a custom-designed waveform generator capable of delivering stimulating signals with unique frequencies and waveforms to as many as eight pairs of electrodes and a joint motion sensor (Mutoh Engineering Inc; Tokyo, Japan) that triggers stimulation of the antagonist once it senses the initiation of an agonist's volitional contraction [24]. Pairs of $3 \times 6 \mathrm{~cm}$ low impedance gel-coated silver fiber electrodes (Nihon Medix Co, 315-1, Mukai-machi, Minami-hanashima, Matsudo-shi; Chiba-ken, Japan) were placed over each motor point of the quadriceps and hamstrings with a

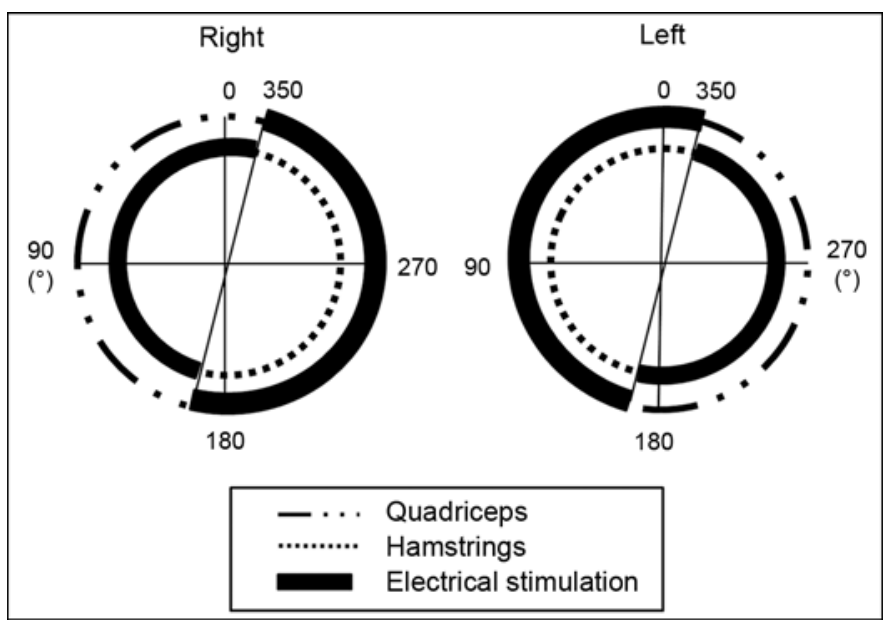

Figure 2.

Protocol of electrical stimulation during cycling exercise with hybrid training system. Position of left pedal was highest at $0^{\circ}$. Position of saddle was inclined to $10^{\circ}$ backward from vertical. Subject sat on cycle ergometer, with his hamstrings electrically stimulated as he volitionally extended his knee and his quadriceps electrically stimulated as he volitionally flexed his knee. Timing of electrical stimulation was controlled by joint motion sensor attached to knee. detector (Top Co, 19-10, Senjyunakai-machi, Adachi-ku; Tokyo, Japan) (Figure 4).

\section{Stimulation Parameters}

The stimulation waveform used in this study consists of a $5,000 \mathrm{~Hz}$ carrier frequency with a pulse width of $200 \mu \mathrm{s}$ modulated at $40 \mathrm{~Hz}$ (2.4 ms on, $22.6 \mathrm{~ms}$ off) to deliver a rectangular biphasic pulse [10]. The electrical stimulator gives constant voltage stimulus to the human body (regulated voltage). It has a stimulus pattern with interlock and limiter for safety. Therefore, the effective current is interlocked at $20 \mathrm{~mA}$ and the peak voltage and current is limited under $72 \mathrm{~V}$ and $90 \mathrm{~mA}$. Stimulation intensities were determined $1 \mathrm{wk}$ before the evaluation session began. We regulated stimulation intensity so that the exercise intensities were adjusted to 80 percent of the maximum comfortable intensity that successfully improved muscle strength and mass without causing pain or numbness $[9,11]$. The mean \pm SD stimulating voltages were $34.5 \pm$ $11.1 \mathrm{~V}$ and $32.9 \pm 10.1 \mathrm{~V}$ for the quadriceps femoris and hamstring muscles, respectively. At these electrical stimulation intensities, all subjects were able to pedal.

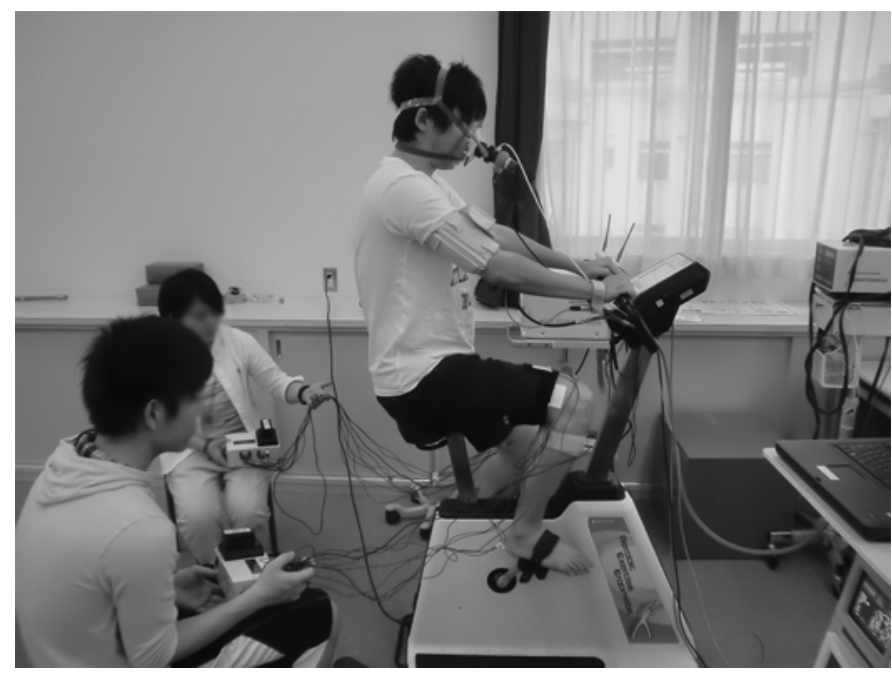

Figure 3.

Cycling exercise with hybrid training system. Electric stimulation was controlled by knee joint motion sensor. Hamstring muscle, which is antagonist of knee extension, was stimulated during knee extension, and quadriceps muscle, which is antagonist of knee flexion, was stimulated during knee flexion. Subjects pedaled cycle ergometer against force generated by electrically stimulated antagonist. 


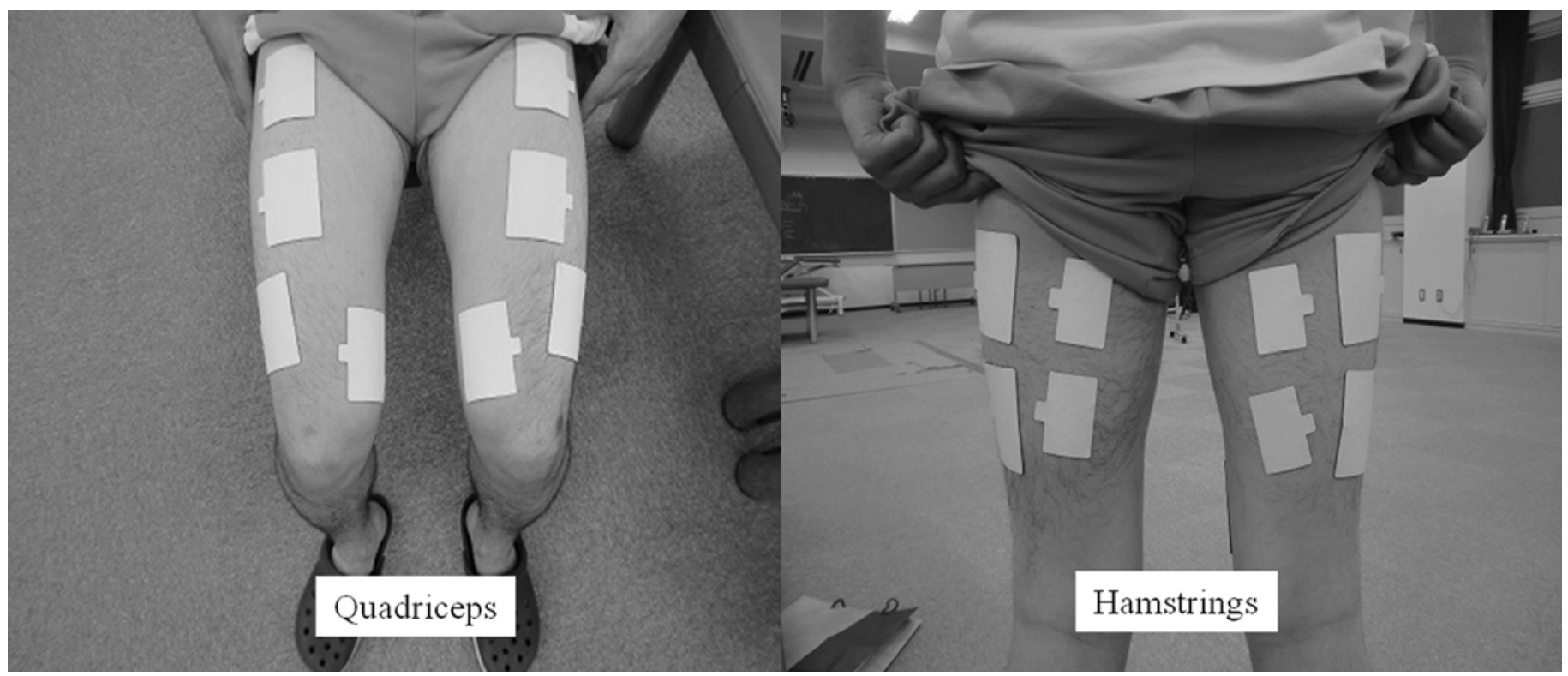

Figure 4.

Surface electrodes. Gel-coated silver fiber electrodes are placed over each motor point of quadriceps and hamstrings.

\section{Statistical Analysis}

All variables are presented as mean $\pm \mathrm{SD}$. Values for $\mathrm{HR}$, absolute $\dot{\mathrm{VO}}_{2}$, relative, $\dot{\mathrm{V}} \mathrm{O}_{2}, \dot{\mathrm{V}} \mathrm{CO}_{2}, \dot{\mathrm{VE}}$, and RER were assessed using a paired $t$-test in order to compare the differences between the HTC test and ULC test for the main purpose of this study. Then, in order to confirm the exercise effects of the HTC test or ULC test compared with a resting condition, a paired $t$-test with Bonferroni correction was used (two comparisons in three tests). All the statistical analyses were performed using JMP Version 9.0 statistical software (SAS Institute Inc; Cary, North Carolina), and $p$-values less than or equal to 0.05 were considered to be statistically significant.

\section{RESULTS}

All subjects completed all the cycling exercise tests without problems, such as not being able to continue pedaling the ergometer from pain or fatigue, although 3 of 12 subjects complained of slight postexercise delayed-onset muscle soreness on the day after the tests.

\section{Ramp Exercise Test}

$\mathrm{VT}$ and $\dot{\mathrm{VO}}_{2 \text { peak }}$ were $20.1 \pm 2.9 \mathrm{~mL} / \mathrm{min}$ and $40.6 \pm$ $4.4 \mathrm{~mL} / \mathrm{min}$, respectively. The workload at VT and
$\dot{\mathrm{V}} \mathrm{O}_{2 \text { peak }}$ were $183.1 \pm 51.6 \mathrm{~W}$ and $321.6 \pm 31.9 \mathrm{~W}$, respectively. HR was $121.6 \pm 9.0 \mathrm{bpm}$ and $181.5 \pm 9.7$ bpm at VT and $\dot{\mathrm{VO}}_{2 \text { peak }}$, respectively.

\section{Physiological Responses to Cycling Exercise}

Results of analysis of expired gas and HR during the cycling exercise tests are identified in the Table. The values of $\mathrm{HR}$, relative $\dot{\mathrm{VO}}_{2}$, absolute $\dot{\mathrm{VO}}_{2}, \dot{\mathrm{V}} \mathrm{CO}_{2}$, and $\dot{\mathrm{VE}}$ were significantly higher during the ULC and HTC tests than during the rest test $(p<0.05)$. Moreover, the values of $\mathrm{HR}$, relative $\dot{\mathrm{VO}} 2$, absolute $\dot{\mathrm{V}}{ }_{2}, \dot{\mathrm{V}} \mathrm{CO}_{2}$, and $\dot{\mathrm{VE}}$ during the HTC test were significantly higher than during the ULC test $(p<0.05)$. However, there were no significant differences in RER between the tests. Absolute $\mathrm{VO}_{2}$ was about $16.7 \pm 2.1$ percent of $\dot{\mathrm{VO}}_{2 \text { peak }}$ during the ULC test and about $21.1 \pm 3.4$ percent during the HTC test.

\section{DISCUSSION}

This is the first report of a cycling exercise utilizing HTS. The findings of this study showed that a cycling exercise with HTS, utilizing electrically stimulated eccentric antagonist muscle contractions as a resistance of motion, increased $\dot{\mathrm{VO}}_{2}$. It showed that the pedaling load was increased by ES applied to antagonist muscles, 
Table.

Analysis of expired gas and heart rate (HR) during two exercise tests: unloaded cycling (ULC) and hybrid training system cycling (HTC) ( $n=12$ subjects). Values are mean \pm standard deviation.

\begin{tabular}{lccc}
\hline Measure & Rest & ULC & HTC \\
\hline$\dot{\mathrm{V}} \mathrm{O}_{2} / \mathrm{kg}(\mathrm{mL} / \mathrm{kg} / \mathrm{min})$ & $4.1 \pm 0.4$ & $6.7 \pm 0.7^{*}$ & $8.6 \pm 1.6^{* \dot{\dagger}}$ \\
$\dot{\mathrm{VO}}{ }_{2}(\mathrm{~mL} / \mathrm{min})$ & $278.6 \pm 30.8$ & $435 \pm 72.6^{*}$ & $555.7 \pm 139.2^{* \dagger}$ \\
$\dot{\mathrm{V} C O}(\mathrm{~mL} / \mathrm{min})$ & $252 \pm 41.2$ & $375.7 \pm 84.3^{*}$ & $487.2 \pm 147^{* \dagger}$ \\
$\dot{\mathrm{V}}(\mathrm{L} / \mathrm{min})$ & $10.1 \pm 1.0$ & $14.1 \pm 2.4^{*}$ & $16.9 \pm 4.2^{* \dagger}$ \\
$\mathrm{RER}$ & $0.91 \pm 0.09$ & $0.86 \pm 0.09$ & $0.86 \pm 0.08$ \\
$\mathrm{HR}(\mathrm{bpm})$ & $76.2 \pm 8.7$ & $93.1 \pm 10.9^{*}$ & $99.1 \pm 9.5^{* \dagger}$ \\
\hline Note: Significant differences between ULC and HTC were determined by paired $t$-test, and significant differences between rest test and ULC or HTC were deter- \\
mined by paired $t$-test with Bonferroni correction (two comparisons in three tests). \\
${ }^{*}$ Bonferroni-adjusted $p<0.05$.
\end{tabular}

and HTS augmented the aerobic energy metabolism during cycling exercise. HTS may be useful as a resistance of motion increasing metabolic cost.

In this study, the cycle ergometer with HTS (HTC) could increase $\mathrm{VO}_{2}$ more than the unloaded cycle ergometer (ULC). Moreover, $\dot{\mathrm{V} C O}{ }_{2}, \dot{\mathrm{V}}$, and $\mathrm{HR}$ showed a similar increase to ULC. These results seem to show that HTS could provide a workload (exercise resistance) for the cycle ergometer without an external resistance source (e.g., resistance using the magnet). HTS is considered applicable to cases in which exercise is limited, such as during space flight or for bedridden patients, because it is compact and portable. Furthermore, HTS seems to be effective for people with limited exercise intensity, such as those with heart disorders or who are elderly, because the exercise intensity of HTC was only about 21 percent of $\dot{\mathrm{V}} \mathrm{O}_{2 \text { peak }}$ below VT. Alternatively, adding physiological exercise resistance to conventional cycle ergometer training would be desirable. These results of cardiopulmonary exercise tests were for sedentary subjects, and physiological responses to HTS will be different in subjects with varying muscular strength and physical fitness.

A potential advantage of HTS is its use of electrically stimulated eccentric contractions [8]. Electrically stimulated eccentric contractions provide 30-50 percent greater force than when using identical stimulation intensity to that of concentric or isometric contractions [25]. In NMES, maximizing force production is commonly necessary to maximize strength gains. However, in electrically stimulated eccentric contractions, even if stimulation intensity is low, relatively major muscular tension can be obtained. In this study, the stimulation intensity used for HTS, which is set according to 80 percent of the maximum comfortable intensity (without pain and discomfort), is lower than the stimulation intensity generally used for NMES, in which discomfort is sometimes reported [26]. Furthermore, electrically stimulated contractions during cycling tend to be much shorter in duration than during NMES for sports injuries, which plays a role in the discomfort and buildup of force. Nevertheless, $\mathrm{V}_{2}$ during $\mathrm{HTC}$ increased more than during ULC because HTS could enhance muscle activity sufficiently by using electrically stimulated eccentric antagonist muscle contractions as a resistance to voluntary agonist muscle contractions (as a substitute for an external braking force). Physiological costs of eccentric contraction are different from concentric contractions, because the recruitment pattern of eccentric exercise is different from that of an equivalent concentric workload [27]. It is reported that $\mathrm{O}_{2}$ cost per unit of work in eccentric cycle exercise is much less than in concentric cycle exercise, but there is a similar positive relationship between $\mathrm{O}_{2}$ cost and workload [17,19]. Bigland-Ritchie and Woods reported that the $\dot{\mathrm{VO}}_{2}$ of submaximal eccentric cycle exercise was approximately one-sixth of the same workload on a motorized bicycle ergometer at $50 \mathrm{rpm}$ [28]. Increased metabolic cost, which is caused by electrically stimulated eccentric contraction of antagonist muscle, is considered small during HTC. However, whether the same metabolic principles of volitional eccentric exercise apply to electrically stimulated eccentric contractions is not known. It is necessary to analyze expired gas during electrically stimulated eccentric exercise without VC.

Another potential advantage of HTS is that it has not only ES but also VC. ES and VC constitute different modes of muscle activation and induce different acute 
physiological effects on the neuromuscular system [7]. For example, ES only stimulates the muscle on which the electrodes are placed in synchrony, whereas VC implies activation from several synergic and stabilizer muscles, which are not stimulated by ES [6-7]. In HTS, by performing ES and VC alternately, VC would activate muscles that are not stimulated by ES. Furthermore, Vanderthommen et al. observed that after a low-intensity intermittent exercise corresponding to 10 percent maximal voluntary torque, ES acidifies more cytoplasm than VC [29]. Therefore, ES strongly activates anaerobic glycolysis. In this study, $\mathrm{VO}_{2}$ during HTC increased more than during ULC although HTS used ES. The reason seems to be that HTS has not only ES but also VC. From the results of this study, we cannot determine the difference in metabolic cost between $\mathrm{ES}$ and $\mathrm{VC}$; however, HTS may have characteristics of both aerobic exercise and anaerobic exercise. Further investigation is necessary to determine the respective metabolic cost of ES and VC during HTS. It may be useful to compare an analysis of expired gas during passive electrically stimulated eccentric cycling exercise without $\mathrm{VC}$ in conditions similar to this study.

In previous studies, HTS had successfully improved not only muscle strength but also muscle mass in healthy men as a resistance exercise when used for flexion exercises of the elbow or knee [8-10]. HTS seems to take advantage of both resistance exercise (anaerobic exercise) by electrical eccentric contractions and aerobic exercise at a low exercise intensity by voluntary concentric contractions. Therefore, HTS may improve not only muscle strength but also physical ability (aerobic power). It is necessary to further evaluate improvements in functional and/or physical ability in addition to muscle strength and/or mass augmentation effect by long-term training using HTS in a larger sample size.

\section{CONCLUSIONS}

These findings indicate that the combined application of VC and EC could lead to a greater increase in metabolic cost. HTS seems to be applicable as a resistance of motion.

\section{ACKNOWLEDGMENTS}

\section{Author Contributions:}

Writing of manuscript: H. Matsuse, N. Shiba, Y. Takano, S. Yamada, H. Ohshima, Y. Tagawa.

Concept/project design (including development of HTC): H. Matsuse, N. Shiba.

Measurement and calculation of data: Y. Takano.

Consultation: S. Yamada, H. Ohshima.

Development of HTC device: Y. Tagawa.

Financial Disclosures: The authors have declared that no competing interests exist.

Funding/Support: This material was based on work supported by a ground-based research grant from Japan Space Exploration Agency and Japan Space Forum (20500489, 20360118).

Institutional Review: The study was designed in accordance with the ethical standards of the Helsinki Declaration of 1975 and received the approval of the Ethics Committee of Kurume University and the Japan Aerospace Exploration Agency. All procedures were fully explained to the participants who gave their written informed consent to participate. Participant Follow-Up: The authors plan to inform participants of the publication of this study.

\section{REFERENCES}

1. Balogum JA, Onilari OO, Akeju OA, Marzouk DK. High voltage electrical stimulation in the augmentation of muscle strength: effects of pulse frequency. Arch Phys Med Rehabil. 1993;74:910-16. [PMID:8379835]

2. Delitto A, Rose SJ, McKowen JM, Lehman RC, Thomas JA, Shively RA. Electrical stimulation versus voluntary exercise in strengthening thigh musculature after anterior cruciate ligament surgery. Phys Ther. 1988;68(5):660-63. [PMID:3258994]

3. Kagaya H, Shimada Y, Ebata K, Sato M, Sato K, Yukawa T, Obinata G. Restoration and analysis of standing-up in complete paraplegia utilizing functional electrical stimulation. Arch Phys Med Rehabil. 1995;76(9):876-81. [PMID:7668962] http://dx.doi.org/10.1016/S0003-9993(95)80556-7

4. Snyder-Mackler L, Delitto A, Bailey SL, Stralka SW. Strength of the quadriceps femoris muscle and functional recovery after reconstruction of the anterior cruciate ligament. A prospective, randomized clinical trial of electrical stimulation. J Bone Joint Surg Am. 1995;77(8):1166-73. [PMID:7642660]

5. Stein RB, Momose K, Bobet J. Biomechanics of human quadriceps muscles during electrical stimulation. J Biomech. 1999;32(4):347-57. [PMID:10213025] http://dx.doi.org/10.1016/S0021-9290(98)00187-0

6. Dehail P, Duclos C, Barat M. Electrical stimulation and muscle strengthening. Ann Readapt Med Phys. 2008;51(6): 
441-51. [PMID:18602713]

http://dx.doi.org/10.1016/j.annrmp.2008.05.001

7. Paillard T. Combined application of neuromuscular electrical stimulation and voluntary muscular contractions. Sports Med. 2008;38(2):161-77. [PMID:18201117] http://dx.doi.org/10.2165/00007256-200838020-00005

8. Yanagi T, Shiba N, Maeda T, Iwasa K, Umezu Y, Tagawa Y, Matsuo S, Nagata K, Yamamoto T, Basford JR. Agonist contractions against electrically stimulated antagonists. Arch Phys Med Rehabil. 2003;84(6):843-48.

[PMID:12808536] http://dx.doi.org/10.1016/S0003-9993(02)04948-1

9. Matsuse H, Shiba N, Umezu Y, Nago T, Tagawa Y, Kakuma T, Nagata K, Basford JR. Muscle training by means of combined electrical stimulation and volitional contraction. Aviat Space Environ Med. 2006;77(6):581-85. [PMID:16780234]

10. Iwasaki T, Shiba N, Matsuse H, Nago T, Umezu Y, Tagawa Y, Nagata K, Basford JR. Improvement in knee extension strength through training by means of combined electrical stimulation and voluntary muscle contraction. Tohoku J Exp Med. 2006;209(1):33-40. [PMID:16636520] http://dx.doi.org/10.1620/tjem.209.33

11. Takano Y, Haneda Y, Maeda T, Sakai Y, Matsuse H, Kawaguchi $T$, Tagawa $Y$, Shiba N. Increasing muscle strength and mass of thigh in elderly people with the hybrid-training method of electrical stimulation and volitional contraction. Tohoku J Exp Med. 2010;221(1):77-85. [PMID:20453461] http://dx.doi.org/10.1620/tjem.221.77

12. Poole DC, Richardson RS. Determinants of oxygen uptake. Implications for exercise testing. Sports Med. 1997;24(5): 308-20. [PMID:9368277] http://dx.doi.org/10.2165/00007256-199724050-00003

13. Xu F, Rhodes EC. Oxygen uptake kinetics during exercise. Sports Med. 1999;27(5):313-27. [PMID:10368878] http://dx.doi.org/10.2165/00007256-199927050-00003

14. Hayter TL, Coombes JS, Knez WL, Brancato TL. Effects of electrical muscle stimulation on oxygen consumption. J Strength Cond Res. 2005;19(1):98-101. [PMID:15705053]

15. Hettinga DM, Andrews BJ. Oxygen consumption during functional electrical stimulation-assisted exercise in persons with spinal cord injury: implications for fitness and health. Sports Med. 2008;38(10):825-38.

[PMID:18803435]

http://dx.doi.org/10.2165/00007256-200838100-00003

16. Arts FJ, Kuipers H. The relation between power output, oxygen uptake and heart rate in male athletes. Int J Sports Med. 1994;15(5):228-31. [PMID:7960315] http://dx.doi.org/10.1055/s-2007-1021051

17. Knuttgen HG, Petersen FB, Klausen K. Oxygen uptake and heart rate responses to exercise performed with concentric and eccentric muscle contractions. Med Sci Sports. 1971;3(1):1-5. [PMID:5095331]

18. Hooker SP, Scremin AM, Mutton DL, Kunkel CF, Cagle G. Peak and submaximal physiologic responses following electrical stimulation leg cycle ergometer training. J Rehabil Res Dev. 1995;32(4):361-66. [PMID:8770800]

19. Perrey S, Betik A, Candau R, Rouillon JD, Hughson RL. Comparison of oxygen uptake kinetics during concentric and eccentric cycle exercise. J Appl Physiol. 2001;91(5): 2135-42. [PMID:11641354]

20. MacKenzie SJ, Rannelli LA, Yurchevich JJ. Neuromuscular adaptations following antagonist resisted training. J Strength Cond Res. 2010;24(1):156-64. [PMID:19996784]

http://dx.doi.org/10.1519/JSC.0b013e3181bd4317

21. Myers J, Bellin D. Ramp exercise protocols for clinical and cardiopulmonary exercise testing. Sports Med. 2000;30(1): 23-29. [PMID:10907755]

http://dx.doi.org/10.2165/00007256-200030010-00003

22. Beaver WL, Wasserman K, Whipp BJ. A new method for detecting anaerobic threshold by gas exchange. J Appl Physiol. 1986;60(6):2020-27. [PMID:3087938]

23. Gaesser GA, Poole DC. The slow component of oxygen uptake kinetics in humans. Exerc Sport Sci Rev. 1996;24: 35-71. [PMID:8744246]

http://dx.doi.org/10.1249/00003677-199600240-00004

24. Yoshimitsu K, Shiba N, Matsuse H, Takano Y, Matsugaki T, Inada T, Tagawa Y, Nagata K. Development of a training method for weightless environment using both electrical stimulation and voluntary muscle contraction. Tohoku J Exp Med. 2010;220(1):83-93. [PMID:20046056] http://dx.doi.org/10.1620/tjem.220.83

25. Seger JY, Thorstensson A. Electrically evoked eccentric and concentric torque-velocity relationships in human knee extensor muscles. Acta Physiol Scand. 2000;169(1):63-69. [PMID:10759612] http://dx.doi.org/10.1046/j.1365-201x.2000.00694.x

26. Lake DA. Neuromuscular electrical stimulation. An overview and its application in the treatment of sports injuries. Sports Med. 1992;13(5):320-36. [PMID:1565927] http://dx.doi.org/10.2165/00007256-199213050-00003

27. Enoka RM. Eccentric contractions require unique activation strategies by the nervous system. J Appl Physiol. 1996;81(6):2339-46. [PMID:9018476]

28. Bigland-Ritchie B, Woods JJ. Integrated electromyogram and oxygen uptake during positive and negative work. J Physiol. 1976;260(2):267-77.[PMID:978517]

29. Vanderthommen M, Duteil S, Wary C, Raynaud JS, LeroyWillig A, Crielaard JM, Carlier PG. A comparison of voluntary and electrically induced contractions by interleaved 1H- and 31P-NMRS in humans. J Appl Physiol. 2003; 94(3):1012-24.[PMID:12571132] 
Submitted for publication April 16, 2012. Accepted in revised form October 16, 2012.

This article and any supplementary materials should be cited as follows:

Matsuse H, Shiba N, Takano Y, Yamada S, Ohshima H, Tagawa Y. Cycling exercise to resist electrically stimu- lated antagonist increases oxygen uptake in males: Pilot study. J Rehabil Res Dev. 2013;50(4):545-54. http://dx.doi.org/10.1682/JRRD.2012.04.0067

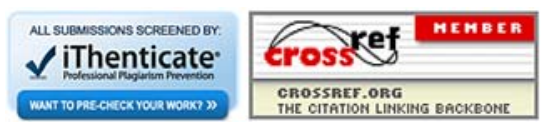


